Immune checkpoint inhibitor-associated polymyalgia rheumatica/giant cell arteritis occurring in a patient after treatment with Nivolumab: a case report

Running Head: Nivolumab-induced large vessel vasculitis

Authors: A. Betrains, $\mathrm{MD}^{1}$; D. Blockmans, $\mathrm{MD}, \mathrm{PhD}^{2}$.

Institutional affiliation:

1. Clinical Department of Internal Medicine, University Hospitals Leuven, Herestraat 49, 3000 Leuven, Belgium. 目

2. Clinical Department of General Internal Medicine, University Hospitals Leuven Research Department of Microbiology and Immunology, Laboratory for Clinical Infectious and Inflammatory Disorders, KU Leuven, Herestraat 49, 3000 Leuven, Belgium.?

Financial support: none

Corresponding author at / requests for reprints at:

Daniel Blockmans, MD, PhD

Department of General Internal Medicine

University Hospitals Leuven

Herestraat 49

B-3000 Leuven, Belgium

Tel.: +32 16344275

E-mail address: daniel.blockmans@uzleuven.be

Word Count: 481

Key Terms: Polymyalgia Rheumatica - Giant Cell Arteritis - Nivolumab - Programmed Cell Death 1 Receptor - Melanoma 


\section{Immune checkpoint inhibitor-associated polymyalgia rheumatica/giant cell arteritis occurring in a patient after treatment with Nivolumab: a case report}

\section{Introduction}

We report a case of polymyalgia rheumatica and giant cell arteritis (PMR/GCA) occurring in a patient with malignant melanoma who received nivolumab therapy, an immunopotentiating antagonist of PD-1.

\section{Case history}

A 72-year-old woman was referred to our clinic with a 3-month history of neck, shoulder and hip myalgias. Recently she developped frontal headache, temporal artery tenderness, jaw claudication and transient blurry vision. Treatment with Nivolumab at a dosage of $3 \mathrm{mg} / \mathrm{kg}$ every 4 weeks was initiated for stage IV malignant melanoma. At the time of diagnosis, she had received 30 treatment cycles. Laboratory studies showed an erythrocyte sedimentation rate (ESR) of $25 \mathrm{~mm} /$ hour and a C-reactive protein of $7 \mathrm{mg} /$ liter. The diagnosis of PMR/GCA was considered, in spite of minimal systemic inflammation, because of pain and stiffness in shoulder and hip girdle combined with characteristic features of GCA, including frontal headache, tenderness of the temporal artery and jaw claudication. 18F-FDG PET/CT findings were consistent with PMR and showed evidence of large vessel vasculitis (fig. 1). Temporal artery biopsy was performed. Histologic examination revealed intima hyperplasia, presence of multinucleated giant cells and lymphocytic infiltration of the tunica intima and media, conforming the diagnosis of GCA. High dose prednisolon was initiated and led to rapid resolution of symptoms.

\section{Discussion}

The immune checkpoint protein programmed cell death protein 1 (PD-1) is a receptor expressed on the surface of cytotoxic T-cells that interacts with the ligand programmed death ligand-1 (PDL-1), which helps the cancer cell evade T-cell-mediated death. Nivolumab is an immune checkpoint inhibitor that prevents the receptor and ligand from binding to each other, thereby disrupting signaling and disinhibiting T cells, which results in an enhanced antitumor immunologic response. ${ }^{1}$ Treatment with nivolumab is also associated with unique immune-related adverse effects (irAEs), including colitis, hepatitis and pneumonitis. ${ }^{2}$ In addition, development of PMR/GCA in patients treated with the CTLA-4 inhibitor ipilimumab, a different type of immune checkpoint inhibitor, was recently described. ${ }^{3}$

Weyand et al investigated the role of PD-1 and PD-L1 interaction in the pathophysiology of arterial wall inflammation in patients with GCA. ${ }^{4}$ Dendritic cells from GCA patients had low PD-L1 expression, whereas the majority of vasculitic T cells expressed PD-1, suggesting inefficiency of the immunoprotective PD-1/PD-L1 immune checkpoint and resembling conditions during anti-PD-1 treatment with nivolumab. ${ }^{4,5}$ Furthermore, Micaily et al recently presented a case of GCA in a 88-year-old female who received pembrolizumab, a PD-1 antagonist similar to Nivolumab, for stage IV non-small-cell lung cancer. ${ }^{6}$ 
As a result of the pathophysiological importance of the PD-1/PD-L1 immune checkpoint in the arterial wall of GCA patients and the recent description of case reports of PMR/GCA ensuing PD-1 inhibitor therapy, we propose the possibility of PMR/GCA as an irAE of antiPD1 treatment. We acknowledge the high prevalence of both conditions and the requirement of a multicenter cohort study to endorse a higher incidence of PMR/GCA during anti-PD1 treatment. On the assumption our hypothesis is confirmed, further research will be necessary to determine a possible therapeutic role of an agent restoring the immunoprotective PD-1/PD-L1 immune checkpoint in the arterial vessel wall of GCA patients. 


\section{References}

1. Larkin, J. et al. Combined nivolumab and ipilimumab or monotherapy in untreated melanoma. N. Engl. J. Med. 373, 23-34 (2015).

2. Friedman CF, Proverbs-Singh TA, Postow MA. Treatment of the Immune-Related Adverse Effects of Immune Checkpoint InhibitorsA Review. JAMA Oncol. 2016;2(10):1346-1353.

3. Goldstein BL, Gedmintas L, Derrick JT. Drug-associated polymyalgia rheumatica/giant cell arteritis occurring in two patients after treatment with ipilimumab, an antagonist of CTLA-4. Arthritis \& rheumatology. 2014;66(3):768-769.

4. Weyand CM, Berry GJ, Goronzy JJ. The immunoinhibitory PD-1/PD-L1 pathway in inflammatory blood vessel disease. J Leukoc Biol. 2018;103(3):565-575.

5. Zhanga $\mathrm{H}$, Watanabea R, Berry GJ et al. Immunoinhibitory checkpoint deficiency in medium and large vessel vasculitis. Proc Natl Acad Sci USA. 2017;114(6):970-979.

6. Micaily I, Chernoff M. An unknown reaction to pembrolizumab: giant cell arteritis. Annals of Oncology. 2017; 28(10):2621-2622. 


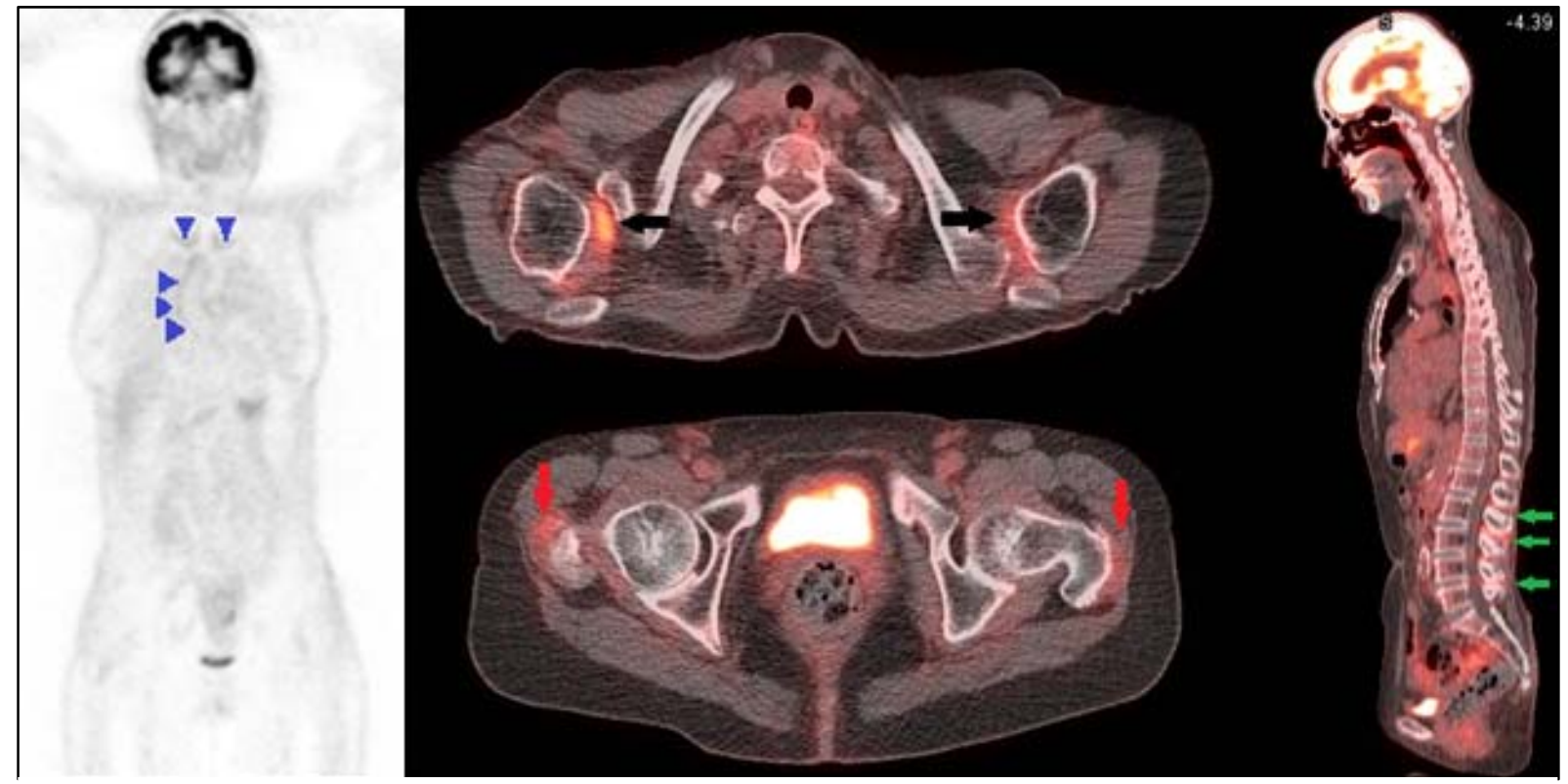

Figure 1: (A) PET examination showing thoracic aortitis, vasculitis of the carotid and subclavian arteries (blue arrowhead); (B) CT-PET showing inflammatory involvement of the shoulders (black arrow); (C) CT-PET showing bilateral greater trochanter bursitis (red arrows); (D) interspinous bursitis at the lumbar level (green arrows). 